\title{
The nanostructure of porous cobalt coatings deposited by magnetron sputtering in helium atmosphere
}

\author{
B. Lacroixa, ${ }^{\star}$, V. Godinho, A. Fernández \\ Instituto de Ciencia de Materiales de Sevilla, CSIC - Universidad de Sevilla, Av. \\ Américo Vespucio 49, 41092 Seville, Spain
}

\begin{abstract}
In this work, (scanning) transmission electron microscopy has been used to study the nanostructure of porous cobalt coatings obtained by magnetron sputtering using helium as process gas. This nanostructure consists in closed pores of different nanometric size (about 4 to $20 \mathrm{~nm}$ ) that are distributed all over a nanocrystalline Co matrix and filled with the deposition gas. Spatially resolved electron energy-loss spectroscopy analysis was applied to measure and map, with high lateral resolution, the relevant physical properties (density, pressure and He-K edge shift) of helium trapped inside these individual nanopores, in order to provide new insights about the growth mechanism involved in such systems. In particular, a coefficient of proportionality, $C=0.039 \mathrm{eV} . \mathrm{nm}^{3}$, between the blue shift of the He K-edge and the He density has been found. In addition, very high He densities (10 - 100 at./nm³) and pressures in the gigapascal range $(0.05-5.0 \mathrm{GPa})$ have been measured. The linear
\end{abstract}

\footnotetext{
a Present address: Departamento de Ciencia de los Materiales e Ingeniería Metalúrgica y Química Inorgánica, Universidad de Cádiz, Facultad de Ciencias, 11510, Puerto Real, Spain.

* Corresponding authors:

E-mail address: bertrand.lacroix@uca.es (B. L.), asuncion@icmse.csic.es (A. F.).
} 
dependence of these parameters as a function of the inverse radii obeying to the Laplace-Young law for most of the pores suggests that their formation during the coating's growth takes place in regime of elastic deformation of the Co matrix.

Keywords: EELS, Spectrum imaging, Helium, Nanopores, Cobalt coatings, Magnetron sputtering.

\section{Introduction}

Materials in the solid state are commonly classified either dense or porous. In the latter case, different type of porosity may exist such as channels that extend through the whole matter, holes or cavities. Such materials cover a variety of applications like molecular adsorption, sensing, catalysis, energy storage and conversion, drug delivery, and more. In this context, the development of advanced synthesis methods to obtain materials with controlled porosity structure is a topic of considerable interest for tuning their properties.

In systems containing pores of nanometric dimensions, the investigation of the porosity structure (including size, shape, distribution and chemical composition) is an important issue to understand and improve the fabrication of materials with tailored functionalities. In previous works, it was shown that the optical and mechanical properties of silicon-based coatings $\left(\mathrm{Si}, \mathrm{SiO}_{x} \mathrm{~N}_{\mathrm{y}}\right)$ can be modified by introducing small pores during their bottom-up fabrication by magnetron sputtering (Godinho et al., 2013, 2012). Very recently, advanced analyses on these systems based on spatially resolved electron energy-loss spectroscopy (EELS) have demonstrated that the nanopores are filled with the deposition gas, helium or 
nitrogen (Lacroix et al., 2016; Schierholz et al., 2015). This approach led among others to the fabrication of devices based on multilayer nanoporous silicon coatings with controlled optical properties (Caballero-Hernández et al., 2015) and to the development of solid helium targets for nuclear reactions (Godinho et al., 2016). In the continuity of these works performed on silicon-based layers, our investigations now extend to other systems, in order to verify the possibility to apply this nanostructuration process based on the trapping of the deposition gas to other type of materials like metals. In this paper, we will focus on nanoporous cobalt coatings. The synthesis of Co films with controlled nanostructures is an attractive topic to modify their optical and magnetic properties by monitoring their growth conditions, using for example oblique angle deposition (Schmidt et al., 2009). Here, (scanning) transmission electron microscopy (S)TEM techniques have been employed to characterize in detail the nanostructure produced in cobalt coatings fabricated by magnetron sputtering using a He plasma and to obtain relevant physical properties of the helium trapped inside the nanopores from spatially resolved EELS: spatial distribution, atomic density, K-edge shift, pressure. Such information will provide additional insights about the formation mechanisms of the nanoporosity involved during the deposition.

\section{Experimental details}

\section{Sample preparation}

According to previous studies (Godinho et al., 2013; Schierholz et al., 2015), cobalt nanostructured porous coating was grown by magnetron sputtering using helium as 
deposition gas with a small amount of argon to maintain the plasma discharge. The deposition was performed on a (001) silicon substrate at a power of 200W in DC mode and at a chamber pressure of 2.8 $\mathrm{Pa}$.

\section{(S)TEM and EELS characterization}

Conventional transmission electron microscopy and scanning transmission electron microscopy were used to study the microstructure of the coating and the nanopores. The experiments were conducted in a FEI TECNAI F30 microscope operated at 300 $\mathrm{kV}$ at the Laboratory of Nanoscopies and Spectroscopies - LANE at the ICMS institute (Seville, Spain). The STEM-EELS spectrum images were recorded through a GATAN GIF Quantum energy filter over 2048 channels using the following experimental conditions: energy dispersion of $0.05 \mathrm{eV} /$ channel, integration times of 0.05 to $0.07 \mathrm{~s}$ per spectrum, step increments ranging from 0.5 to $1.5 \mathrm{~nm}$, energy resolution of $0.9 \mathrm{eV}$, convergence and collection semi-angles of $12.4 \mathrm{mrad}$ and 9.6 mrad respectively, beam current of the electron probe of about $50 \mathrm{pA}$, and probe size of $0.2 \mathrm{~nm}$. The dwell time was optimized to maximize the He-K signal, keeping at the same time the elastic peak intensity below the saturation level of the CCD detector. At the same time, the high angle annular dark field (HAADF) signal was also acquired to provide a Z-contrast image of the investigated area. TEM crosssections of the coatings were prepared by mechanical polishing followed by ion milling with argon ions up to electron transparency. Just before the TEM observations, the specimen surface was cleaned in an $\mathrm{Ar} / \mathrm{O}_{2}$ plasma in order to remove the hydrocarbon contamination. 


\section{Results}

\section{Overall coating microstructure}

The TEM bright field image shown in Figure 1a presents an overview of the coating where pores of various size (from 4 to $20 \mathrm{~nm}$ approximately) can be observed in bright contrast, homogeneously distributed over the whole layer. These pores exhibit either spherical or facetted shapes (Fig. 1b). The presence of facets has been previously reported in bubbles formed in a variety of ion-irradiated materials depending on the type of implanted atoms and matrix nature (Fleischer and Norton, 1996; Templier, 1991), and it may be attributed to a surface energy minimization of the cavities (Goodhew, 1981; Herring, 1951; Snoeck et al., 2006). The selected area electron diffraction (SAED) pattern recorded in the middle of the coating indicates that the coating structure is polycrystalline. The radial integration profile of the diffraction pattern (Fig. 1c) shows peaks that match with the hexagonal structure of cobalt, which corresponds to its stable form at temperatures below about $400^{\circ} \mathrm{C}$ (Betteridge, 1980). The small discrepancies observed between the profile and the reciprocal plane spacings may be attributed to residual stresses commonly observed in thin films. 

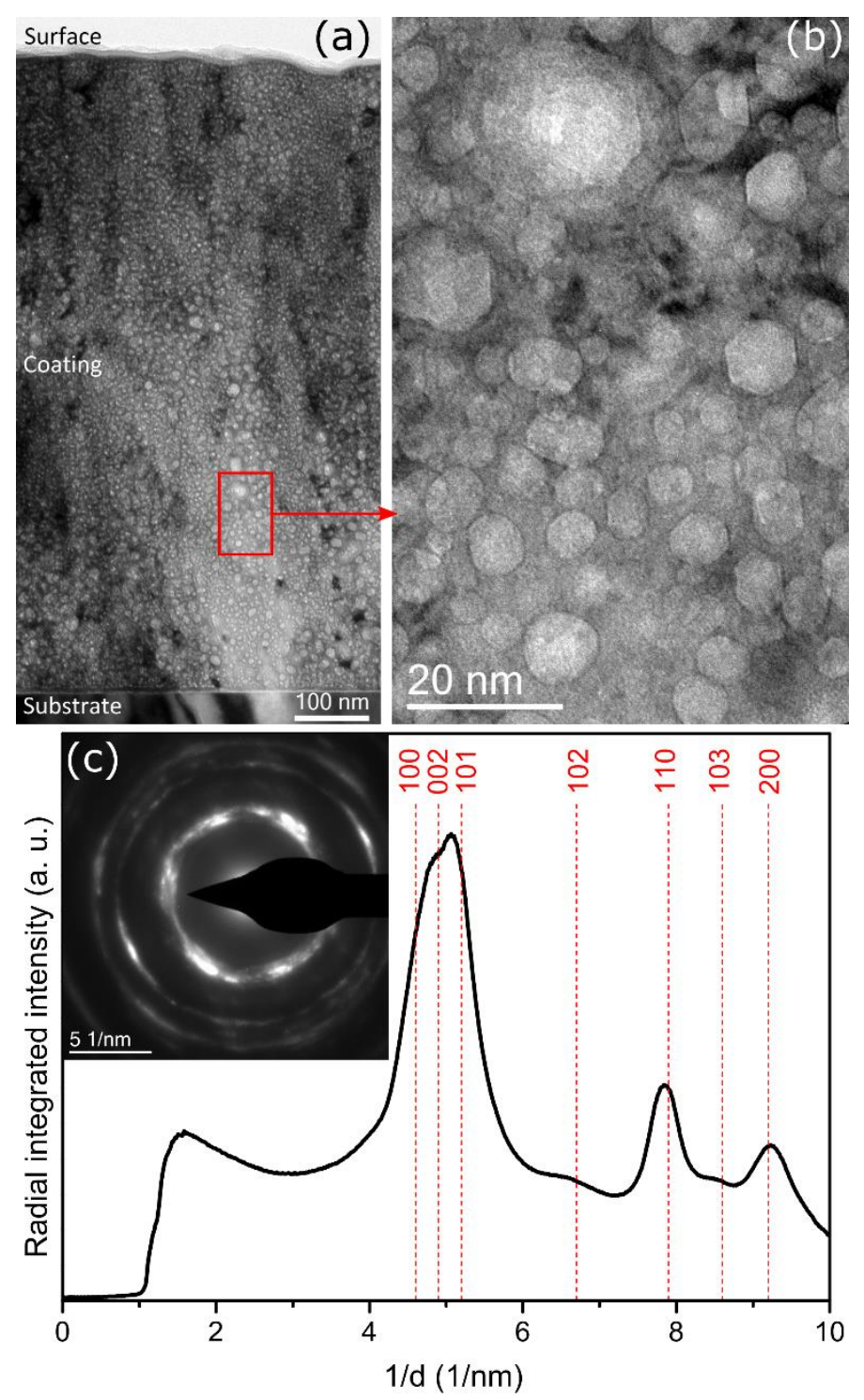

Figure 1: (a) and (b) Bright field TEM images of the Co coating at different magnifications. (c) SAED recorded in the middle of the coating and the corresponding radial integrated intensity profile. The vertical lines indicate the interplanar distances of the hexagonal structure of cobalt.

Analysis of the helium state inside the nanopores by STEM-EELS

In our previous works, similar nanoporous structures have been observed in Sibased coatings grown by magnetron sputtering using $\mathrm{He}$ or $\mathrm{N}$-rich atmospheres. 
Following previous studies on the properties of trapped helium inside small bubbles formed during ion irradiation (David et al., 2014, 2011; Fréchard et al., 2009; McGibbon, 1991; Taverna et al., 2008; Walsh et al., 2000), we have used advanced STEM-EELS analyses to evidence that the nanopores formed during the film deposition are filled with the process gases $\mathrm{He}$ (Schierholz et al., 2015) or $\mathrm{N}_{2}$ (Lacroix et al., 2016) in condensed states. In the present study, the chemical sensitivity and spatial resolution of STEM-EELS have been considered again to determine the He distribution inside cobalt layers and to characterize the properties of individual nanopores. This technique was preferred to the recently developed gentle procedure based on energy-filtered TEM (Alix et al., 2015; Dérès et al., 2017; Seydoux-Guillaume et al., 2016) in order to keep the same approach as in our previous investigations. It also offers better energy resolution and signal-to-noise ratio.

Figure 2 compares two EELS spectra recorded inside and outside a pore which appears in dark contrast on the HAADF image presented later on Fig. 3c. Across the pore region, a sharp peak around $23 \mathrm{eV}$ appears on top of the Co plasmon. This peak, attributed to the $\mathrm{He} 1 \mathrm{~s} \rightarrow 2 \mathrm{p}$ transition, confirms that a significant amount of helium remains trapped inside the cavity. This transition normally occurs at energy of $21.218 \mathrm{eV}$ for free atoms, but shifts towards the higher energies (blue shift) when the He density increases (Rife et al., 1981; Walsh et al., 2000). This phenomenon is attributed to a short-range Pauli repulsion between the electrons of neighboring atoms (Lucas et al., 1983). 


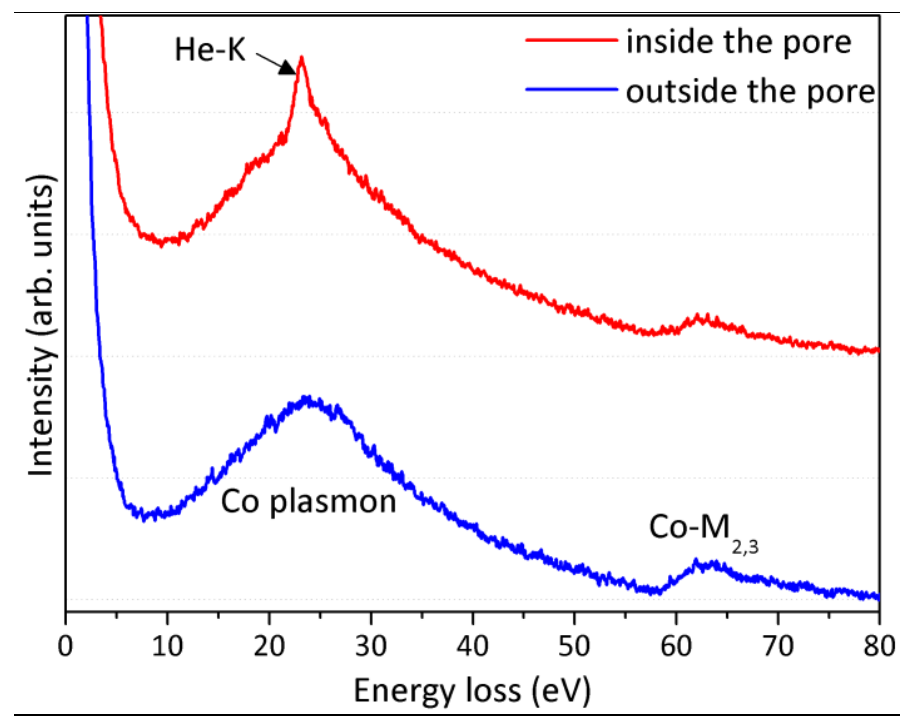

Figure 2: Comparison of low-loss EELS spectra recorded inside and outside one pore: across the pore, an extra peak corresponding to the He-K edge appears on top of the Co plasmon.

In order to obtain quantitative information from the helium signal, we developed our own program running with MATLAB for in-depth analyses of the spectrum image datasets. First, the extraction of the He-K edge from the EELS spectra requires a special care. Before extraction, the dataset was processed using the following procedure. The raw spectra were first aligned with the zero loss peak (ZLP) at the zero energy-loss reference. The statistical noise was then removed using multivariate statistical analysis (MSA) (Bonnet, 1998). Finally, Fourier-log deconvolution was applied to remove the multiple scattering contributions (Egerton, 2011).

To extract the helium signal, the background coming from the Co plasmon of the matrix has to be subtracted properly. For that purpose, different plasmon subtraction procedures may be considered. Using the matrix as reference is a straightforward 
but unreliable option due to the changes in the plasmon shape arising from the projected thickness fluctuations across the cavities. A Drude model is another alternative but its implementation is more complex. For those reasons, a simplest but reliable approach based on the Gaussian fit of the top of the cobalt plasmon was preferred, using two fitting windows on both side of the He peak. The typical fitting ranges are [16.0-21.2] eV and [25.2-30.0] eV, but these ranges may change slightly depending on the position of the He peak. Figure 3a presents examples of plasmon fitting done on different spectra recorded outside, at the border and inside one pore. The corresponding helium signals obtained after subtracting the plasmon functions are shown in Fig. 3b: here, we can notice that the background chosen for the He signal extraction is suitable and does not introduce significant artifacts. Also note that both the main $1 s \rightarrow 2 p$ and the weaker $1 s \rightarrow 3 p$ transitions are visible (at about 23 and $25 \mathrm{eV}$, respectively). The main residual helium signal was then fitted with a Gaussian function (Fig. 3b), and the corresponding integral intensity over 4 eV $\left(I_{\mathrm{He}}\right)$ and energy shift relative to the free atom value $\left(\Delta E_{H e}\right)$ were used to characterize the $\mathrm{He} 1 \mathrm{~s} \rightarrow 2 \mathrm{p}$ transition. This procedure was iterated for each position of the dataset to obtain 2D maps of $I_{H e}$ and $\Delta E_{H e}$ shown in Fig. 3d and 3e. 

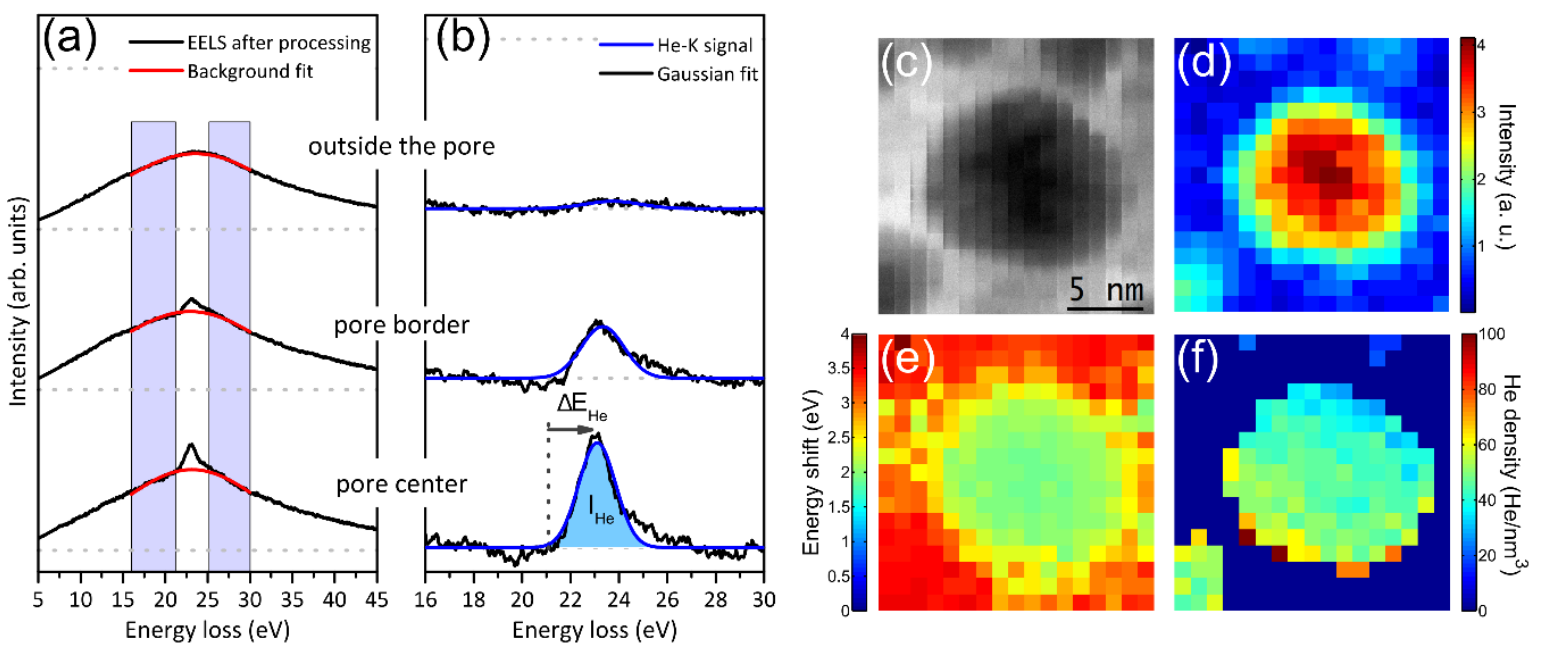

Figure 3: (a) EELS spectra (after processing) recorded at different positions of the spectrum image: outside the pore, at the pore border, and in the pore center. The blue rectangles define the two windows used for fitting the Co plasmon background on both side of the He-K edge. (b) He-K signal obtained after subtraction of the plasmon contribution. (c) STEM-HAADF image recorded over one pore of the coating. (d) He-K intensity map $I_{H e}$. (e) He-K energy shift map $\Delta E_{H e}$. (f) He density $\operatorname{map} n_{H e}$.

The helium density $\mathrm{n}_{H e}$ inside the pores was determined, for each position, from the intensity of the He $1 \mathrm{~s} \rightarrow 2 \mathrm{p}$ transition $I_{H e}$, using the following equation (Walsh et al., 2000):

$$
n_{H e}=\frac{I_{H e}}{I_{Z L P}} \frac{1}{\sigma_{H e} h}(1)
$$

$I_{Z L P}$ represents the integrated intensity of the elastic peak. The angle-integrated cross-section for the $1 \mathrm{~s} \rightarrow 2 \mathrm{p}$ transition, $\sigma_{H e}$, was calculated in our experimental conditions (see Experimental details section), taking into account the corrections for 
the incident beam convergence (for calculation, see the work by Walsh et al. (Walsh et al., 2000)). This parameter was evaluated at the energy corresponding to the maximum of the He-K fitted peak. Typical values of $\sigma_{H e}$ are about $4.8 \times 10^{-24} \mathrm{~m}^{2}$. The parameter $h$ corresponds to the pore thickness crossed by the electron beam: it was determined as the complement to local thickness measurements of the matrix using the log-ratio method (Taverna et al., 2008), considering a mean free path $\lambda=132.5$ $\mathrm{nm}$. The latter was calculated with the lakoubovskii's formula (lakoubovskii et al., 2008), using our experimental conditions (convergence and collection semi-angles, accelerating voltage) as well as the density of the hexagonal phase of cobalt $\rho=$ $8.832 \mathrm{~g} / \mathrm{cm}^{3}$ (Betteridge, 1980) as input parameters.

After applying equation (1) on the whole dataset, a 2D map of the He density was obtained, as presented in Fig. 3f. On this figure, only the pixels having an intensity value higher than $25 \%$ of the maximum He signal intensity were plotted. We can see that the He density determined in the middle of the pore (Fig. 3f) ranges from 40 to 50 at. $/ \mathrm{nm}^{3}$. The fact that $n_{H e}$ and $\Delta E_{H e}$ remain constant across the pore confirms that no helium desorption occurred during measurements (contrary to recent observations done on He nanocavities embedded in Si or Ge (David et al., 2014, 2011; Schierholz et al., 2015)) which is a good indication of its stability under the electron beam. The mean He density and the mean energy shift of the $1 s \rightarrow 2 p$ transition were finally determined over a set of pixels (typically over around 10 pixels) selected in the pore center to limit surface effects (Taverna et al., 2008). For the pore presented in Fig. 3, a mean density $n_{H e}=44$ at. $/ \mathrm{nm}^{3}$ and a mean energy shift $\Delta E_{H e}=1.94 \mathrm{eV}$ were obtained. 
The mean He pressure inside the pores, $P_{H e}$, was then calculated from the density. The choice of a suitable equation of state (EOS) was discussed in detail by Walsh et al. as a function of the temperature and He density range (Walsh et al., 2000). In our case, the Trinkaus EOS (Trinkaus, 1983) was applied as it is considered as the most accurate EOS available in our density range (up to a hundred at./nm ${ }^{3}$ ). For the pressure calculation, we assume that the temperature during the pore formation is $\mathrm{T}=293 \mathrm{~K}$ (growth performed at RT). A mean pressure close to $0.5 \mathrm{GPa}$ was found for the pore shown in Fig. 3.

The EELS analysis presented above was applied to a set of pores of different size (from 4 to $20 \mathrm{~nm}$ ) found in the Co coating. As shown on Fig. 4, a linear relationship between the energy shift of the He $1 \mathrm{~s} \rightarrow 2 \mathrm{p}$ transition and the He density was found, in agreement with previous works of the literature (David et al., 2011; Fréchard et al., 2009; Jäger et al., 1982; McGibbon, 1991; Taverna et al., 2008; Walsh et al., 2000). The values $n_{H e}$ and $\Delta E_{H e}$ in our dataset are approximately in the [10-100] at. $/ \mathrm{nm}^{3}$ and [0.7-4.3] eV ranges, respectively. Our data were fitted linearly and a law of the form $\Delta E_{H e}=C \times n_{H e}+\delta e$, with $C=0.039 \mathrm{eV} \cdot \mathrm{nm}^{3}$ and $\delta e=0.44 \mathrm{eV}$ was found. The $C$ parameter determined here is significantly higher than those found by David et al. ((David et al., 2011) $\left.C=0.015 \mathrm{eV} . \mathrm{nm}^{3}\right)$, Walsh et al. ((Walsh et al., 2000) $\left.C=0.016 \mathrm{eV} \cdot \mathrm{nm}^{3}\right)$, Fréchard et al. ((Fréchard et al., 2009) $C=0.019 \mathrm{eV} \cdot \mathrm{nm}^{3}$ ) or Jäger et al. ((Jäger et al., 1982) $\left.C=0.024 \mathrm{eV} \cdot \mathrm{nm}^{3}\right)$, but is much closer to those found by McGibbon et al. ((McGibbon, 1991) $\left.C=0.035 \mathrm{eV} \cdot \mathrm{nm}^{3}\right)$ or Taverna et al. ((Taverna et al., 2008) $\left.C=0.044 \mathrm{eV} . \mathrm{nm}^{3}\right)$. However, these differences for the $C$ values are not necessarily so significant, since the errors on the density can be large, especially in 
the smallest pores. This uncertainty is mainly attributed to the extraction of projected thickness maps of the cavities, which relies on many assumptions (determination of the total specimen thickness from log-ratio method, choice of a reference thickness of the matrix which is assumed to be constant all around the pore). Fréchard et al. also mentioned that the small number of data points in the previously published work may also explain the different values found for $C$ (Fréchard et al., 2009).

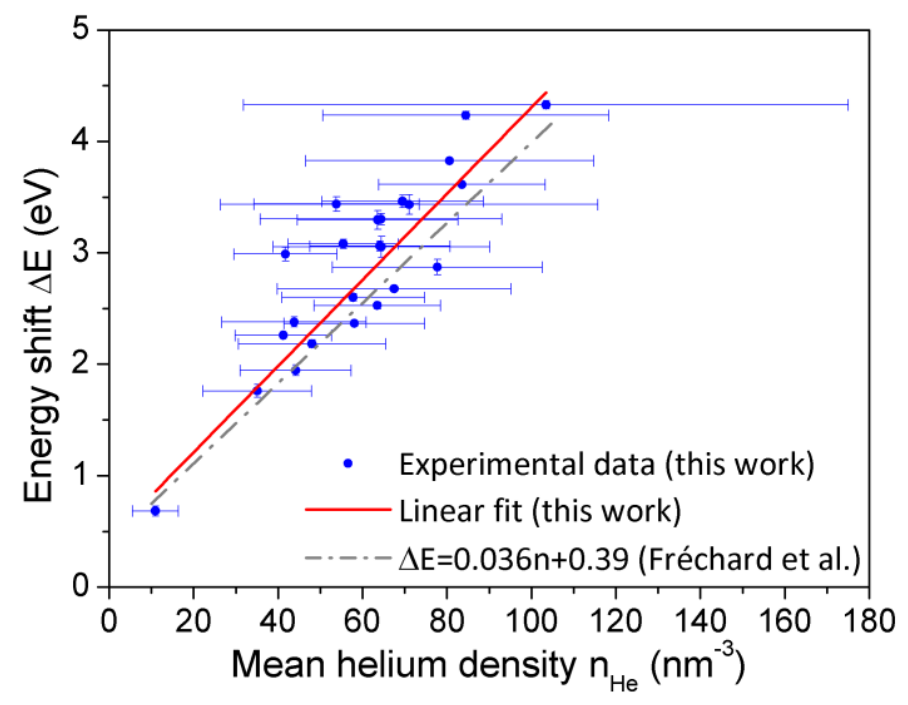

Figure 4: Mean energy shift of the $\mathrm{He} 1 s \rightarrow 2 p$ transition as a function of the mean He density measured in various pores. The red line corresponds to the linear fit of our data set. The dashed grey line is a general law given by Fréchard et al. (Fréchard et al., 2009) that is plotted for comparison. For each point, the error on the mean energy shift was taken as the standard deviation on the set of pixels selected on the energy shift map. The uncertainty on $n_{H e}$ was estimated by error propagation, assuming the errors on $I_{Z L P}$ and $\sigma_{H e}$ are negligible as compared with the errors on $I_{H e}$ (estimated from the residual of the Gaussian fit) and $h$ (standard deviation of the selected pixels in the pore thickness map). 
It is worth to mention that our $\Delta E_{H e}=f\left(n_{H e}\right)$ relation, obtained over a wide range of He density, is very similar to the general law presented in the work of Fréchard et al. $\left(\Delta E_{\mathrm{He}}=0.036 \times n_{\mathrm{He}}+0.39\right)$ that already took into account the results obtained in different previous studies (Fréchard et al., 2009). This is therefore a good indication of the reliability of the methodology we applied to the measurement of the He density in the nanopores of cobalt coatings.

Energy shift, He density and He pressure inside the pores were investigated as a function of the pore dimension. In the present case, the pore size $d$ was estimated as the maximum of the local pore thickness $h$ among the pixels chosen to calculate the mean density (in the pore center). This choice was validated by the measurement of the lateral size of the pores on the HAADF images.

Figure 5 presents the evolution of the energy shift of the He $1 s \rightarrow 2 p$ transition as a function of the pore size obtained in the cobalt coating. As seen on the inset figure, the extracted He-K edges for different pores present a progressive shift towards the higher energies as their size decreases. The mean energy shifts, obtained for a set of pores of size ranging from 4 to $20 \mathrm{~nm}$, were plotted versus their inverse radii $r$ (the latter were estimated as $r=d / 2$, assuming that the pores are spherical). For most of the pores, the $\Delta E_{\mathrm{He}}$ values increase from around 1.7 to $4.3 \mathrm{eV}$ as the pore size decreases. Despite the important spreading of the dataset, an approximate evolution of $\Delta E_{H e}$ versus $1 / r\left(\Delta E_{H e}=5.6 / r+1.5\right)$ was obtained by linear fitting. 


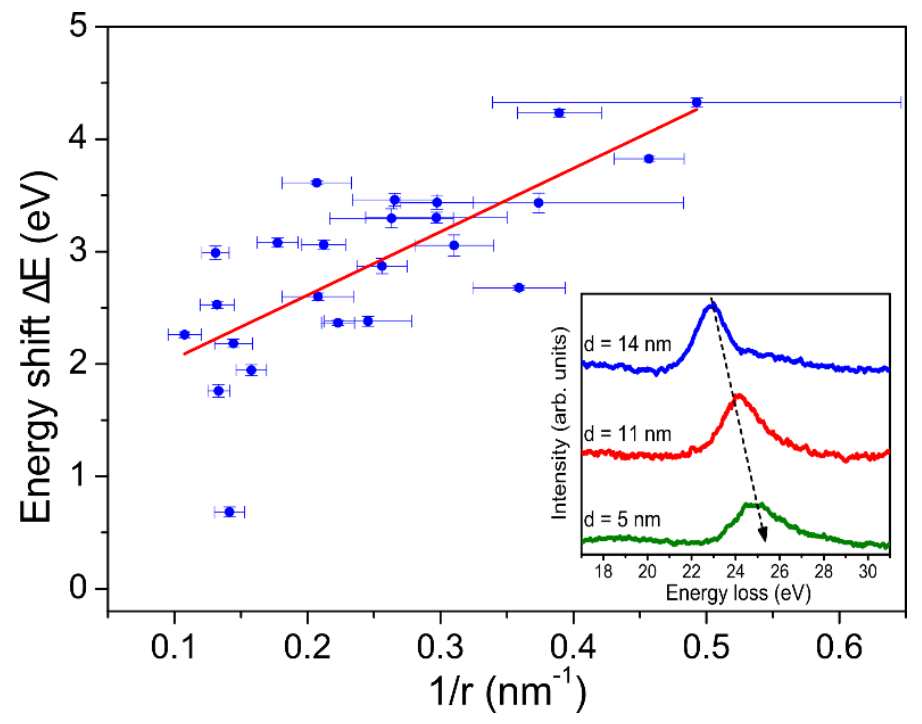

Figure 5: Mean energy shift as a function of the inverse radii of the pores. For each point, the errors on the mean energy shift and on the pore size were taken as the standard deviation on the set of pixels selected on the energy shift map and pore thickness map, respectively. The uncertainties for $1 / r$ were estimated by error propagation. The red line corresponds to the linear fit of the dataset. On the inset are shown some extracted He-K edges for pores of different sizes. The arrow points out the energy shift evolution as the pore size decreases.

Helium density and the pressure measured inside the different investigated pores are plotted on Fig. 6 as a function of their inverse radii. As expected from the plot of Fig. 4, an increase of these two parameters was pointed out as the pore size is reduced. For most of the pores, the density values obtained among the largest and the smallest pores extend roughly from 40 to $100 \mathrm{at} . / \mathrm{nm}^{3}$, respectively, above the density of liquid helium (20 at./nm³ (Jäger et al., 1982)). The fit of these data confirms the linear behavior of $n_{H e}$ versus $1 / r$. The related internal He pressure obtained using the Trinkaus EOS increases linearly from around 0.4 to $5 \mathrm{GPa}$, following a law 
$P_{H e}=5.23 / r+0.07$ according to the fit of our dataset (the point of highest pressure was excluded since it deviates strongly from the other data points). If we consider the surface energy $\gamma=2.704 \mathrm{~J} / \mathrm{m}^{2}$ for polycrystalline cobalt at $273 \mathrm{~K}$ (Betteridge, 1980), this pressure evolution is in very good agreement with the equilibrium pressure given by the Laplace-Young law $P=2 \gamma / r$, suggesting that the pores deforms the matrix elastically (Taverna et al., 2008). Such radius dependence (not observed in covalent materials (Dérès et al., 2017)) has been reported earlier in irradiated metals (Fréchard et al., 2009; Taverna et al., 2008; Walsh et al., 2000), but most of He nanobubbles were found to be under-pressured. In our case, helium pores have been formed by a bottom-up approach during the coating growth process. In previous simulation work, we concluded that for thin films prepared using $\mathrm{He}$, in addition to the shadowing effects typical of physical vapor deposition, a new process considering a certain He mobility during the coating's growth should be responsible for coarsening of small pores into bigger and stable pores (Godinho et al., 2014). Similar behavior was reported for implanted He in metals (Morishita, 2007; Pizzagalli et al., 2013; Wilson et al., 1981). We believe that in our case there is a dynamical process in which by one hand the He atoms get trapped inside the matrix and on the other hand can merge to form bigger pores as the film grows, and this occurs in a regime of elastic deformation of the matrix. After deposition, the formed pores are very stable keeping the gas inside for years (Godinho et al., 2016; Lacroix et al., 2016).

These results give new insights about the growth of Co films by magnetron sputtering using helium atmosphere. First, they reveal that a high amount of He can be trapped inside small pores formed during the deposition process. They also confirm that 
helium atoms remain very stable and do not escape by migration in the matrix. This is corroborated by the small number of pores found in the coatings which did not present any helium signal. Actually, in Fig. 5 and Fig. 6, we can notice that only one measurement deviates significantly from the linear behavior versus $1 / r$ (energy shift around $0.7 \mathrm{eV}$, density around 10 at. $/ \mathrm{nm}^{3}$, pressure around $0.05 \mathrm{GPa}$ ). In comparison to the other pores, this one seems under-pressured, which may be explained by a possible He desorption that occurred before or during its observation.
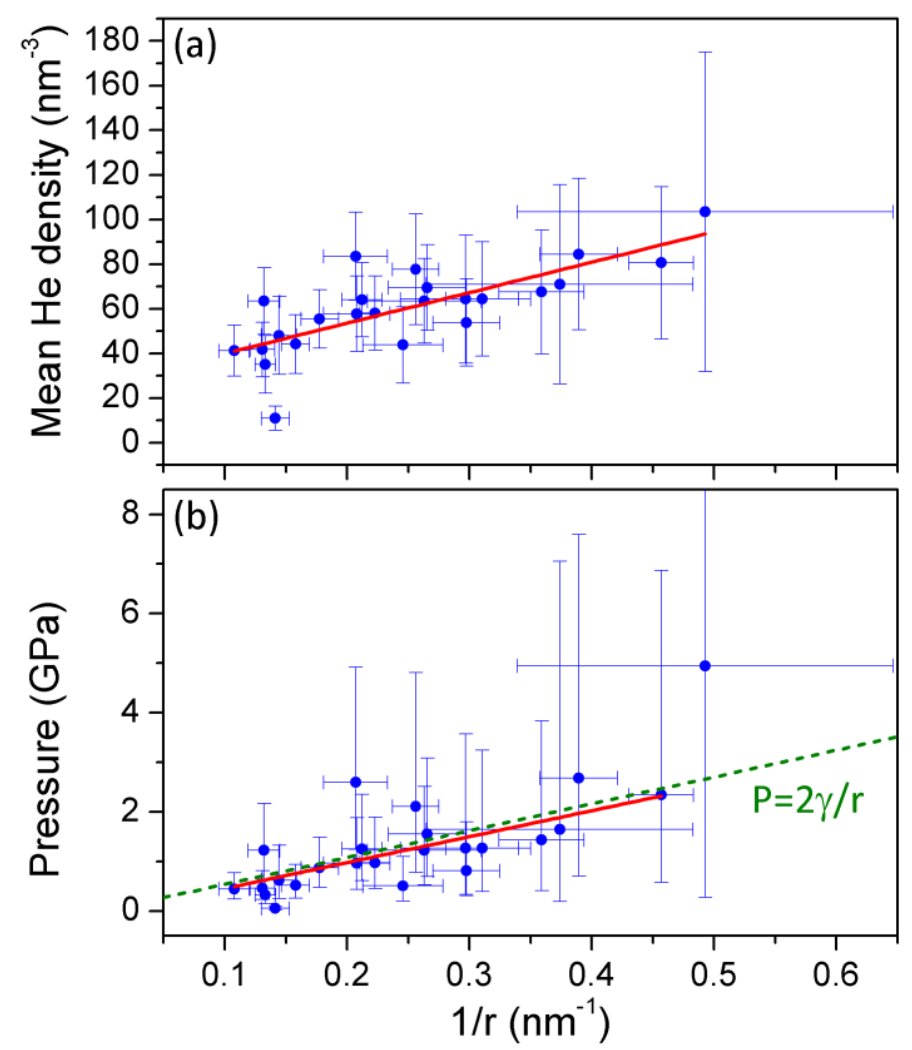

Figure 6: (a) Mean density and (b) mean pressure of He inside the pores as a function of their inverse radii. The red lines correspond to the linear fit of the datasets. The dashed green line on (b) shows the theoretical linear relation for elastic deformation of the cobalt matrix. For each point, the uncertainties for $n_{H e}$ were 
estimated by error propagation. Errors for $P_{H e}$ were determined by calculating the variation of the EOS from the density range defined by density error bars.

\section{Conclusion}

The aim of this work was to characterize porous cobalt coatings grown by magnetron sputtering using helium as process gas. TEM and spatially resolved EELS were used to study the coating's nanostructure including the pore composition and physical properties. It was clearly evidenced that these layers contain closed pores of nanometric size that are filled with the deposition gas $\mathrm{He}$, at high density yielding to gigapascal internal pressure. The extensive analysis of the EELS data recorded for a set of pores allowed to determine a proportionality constant between the energy shift of the He-K edge and the He density, in good agreement with the literature for other systems containing $\mathrm{He}$ nanobubbles. In addition, we found that the $\mathrm{He}$ pressure inside the nanopores corresponds to the expected equilibrium pressure indicating that the pores are stabilized following a regime of elastic deformation of the Co matrix.

\section{Acknowledgement}

This work was supported by the EU 7FP (project AI-NanoFunc CT-REGPOT-20111-285895, http://www.al-nanofunc.eu/), the Spanish Ministry MINECO (project CTQ2015-65918-R), and the CSIC (PIE 201760E002). The authors also acknowledge the Laboratory for Nanoscopies and Spectroscopies for the TEM facilities. 


\section{References}

Alix, K., David, M.-L., Lucas, G., Alexander, D.T.L., Pailloux, F., Hébert, C., Pizzagalli, L., 2015. Gentle quantitative measurement of helium density in nanobubbles in silicon by spectrum imaging. Micron 77, 57-65. https://doi.org/10.1016/j.micron.2015.05.011

Betteridge, W., 1980. The properties of metallic cobalt. Prog. Mater. Sci. 24, 51-142. https://doi.org/10.1016/0079-6425(79)90004-5

Bonnet, N., 1998. Multivariate statistical methods for the analysis of microscope image series: applications in materials science. J. Microsc. 190, 2-18. https://doi.org/10.1046/j.1365-2818.1998.3250876.x

Caballero-Hernández, J., Godinho, V., Lacroix, B., Jiménez De Haro, M.C., Jamon, D., Fernández, A., 2015. Fabrication of optical multilayer devices from porous silicon coatings with closed porosity by magnetron sputtering. ACS Appl. Mater. Interfaces 7, 13889-13897. https://doi.org/10.1021/acsami.5b02356

David, M.-L., Alix, K., Pailloux, F., Mauchamp, V., Couillard, M., Botton, G.A., Pizzagalli, L., 2014. In situ controlled modification of the helium density in single helium-filled nanobubbles. J. Appl. Phys. 115, 123508. https://doi.org/10.1063/1.4869213

David, M.-L., Pailloux, F., Mauchamp, V., Pizzagalli, L., 2011. In situ probing of helium desorption from individual nanobubbles under electron irradiation. Appl. Phys. Lett. 98, 171903. https://doi.org/10.1063/1.3582612

Dérès, J., David, M.-L., Alix, K., Hébert, C., Alexander, D.T.L., Pizzagalli, L., 2017. Properties of helium bubbles in covalent systems at the nanoscale : a combined numerical and experimental study. Phys. Rev. B 96, 14110. https://doi.org/10.1103/PhysRevB.96.014110

Egerton, R.F., 2011. Electron energy-loss spectroscopy in the electron microscope, Third Edit. ed. Springer, Boston, MA. https://doi.org/10.1007/978-1-4419-95834

Fleischer, E.L., Norton, M.G., 1996. Noble gas inclusions in materials. Heterog. Chem. Rev. 3, 171-201. 
Fréchard, S., Walls, M., Kociak, M., Chevalier, J.P., Henry, J., Gorse, D., 2009. Study by EELS of helium bubbles in a martensitic steel. J. Nucl. Mater. 393, 102-107. https://doi.org/10.1016/j.jnucmat.2009.05.011

Godinho, V., Caballero-Hernández, J., Jamon, D., Rojas, T.C., Schierholz, R., García-López, J., Ferrer, F.J., Fernández, A., 2013. A new bottom-up methodology to produce silicon layers with a closed porosity nanostructure and reduced refractive index. Nanotechnology 24, 275604. https://doi.org/10.1088/0957-4484/24/27/275604

Godinho, V., Ferrer, F.J., Fernández, B., Caballero-Hernández, J., GómezCamacho, J., Fernández, A., 2016. Characterization and validation of a-Si magnetron-sputtered thin films as solid He targets with high stability for nuclear reactions. ACS Omega 1, 1229-1238. https://doi.org/10.1021/acsomega.6b00270

Godinho, V., Moskovkin, P., Álvarez, R., Caballero-Hernández, J., Schierholz, R., Bera, B., Demarche, J., Palmero, A., Fernández, A., Lucas, S., 2014. On the formation of the porous structure in nanostructured a-Si coatings deposited by dc magnetron sputtering at oblique angles. Nanotechnology 25, 355705. https://doi.org/10.1088/0957-4484/25/35/355705

Godinho, V., Rojas, T.C., Fernández, A., 2012. Magnetron sputtered a-SiOxNy thin films: a closed porous nanostructure with controlled optical and mechanical properties. Microporous Mesoporous Mater. 149, 142-146. https://doi.org/10.1016/j.micromeso.2011.08.018

Goodhew, P.J., 1981. Shapes of pores in metals. Met. Sci. 15, 377-385. https://doi.org/10.1179/030634581790426868

Herring, C., 1951. Some theorems on the free energies of crystal surfaces. Phys. Rev. 82, 87-93. https://doi.org/10.1103/PhysRev.82.87

lakoubovskii, K., Mitsuishi, K., Nakayama, Y., Furuya, K., 2008. Thickness measurements with electron energy loss spectroscopy. Microsc. Res. Tech. 71, 626-631. https://doi.org/10.1002/jemt.20597

Jäger, W., Manzke, R., Trinkaus, H., Crecelius, G., Zeller, R., Fink, J., Bay, H.L., 1982. Density and pressure of helium in small bubbles in metals. J. Nucl. Mater. 
111-112, 674-680. https://doi.org/10.1016/0022-3115(82)90288-4

Lacroix, B., Godinho, V., Fernández, A., 2016. Nitrogen nanobubbles in a-SiOxNy coatings: evaluation of its physical properties and chemical bonding state by spatially resolved electron energy-loss spectroscopy. J. Phys. Chem. C 120, 5651-5658. https://doi.org/10.1021/acs.jpcc.5b09036

Lucas, A.A., Vigneron, J.P., Donnelly, S.E., Rife, J.C., 1983. Theoretical interpretation of the vacuum ultraviolet reflectance of liquid helium and of the absorption spectra of helium microbubbles in aluminum. Phys. Rev. B 28, 24852496. https://doi.org/10.1103/PhysRevB.28.2485

McGibbon, A.J., 1991. The application of PEELS on a STEM to the problem of inert gas bubbles in solids. Inst. Phys. Conf. Ser. 119, 109-112.

Morishita, K., 2007. Nucleation path of helium bubbles in metals during irradiation. Philos. Mag. 87, 1139-1158. https://doi.org/10.1080/14786430601096910

Pizzagalli, L., David, M.-L., Bertolus, M., 2013. Molecular dynamics simulation of the initial stages of He bubbles formation in silicon. Model. Simul. Mater. Sci. Eng. 21, 65002. https://doi.org/10.1088/0965-0393/21/6/065002

Rife, J.C., Donnelly, S.E., Lucas, A.A., Gilles, J.M., Ritsko, J.J., 1981. Optical absorption and electron-energy-loss spectra of helium microbubbles in aluminum. Phys. Rev. Lett. 46, 1220-1223. https://doi.org/10.1103/PhysRevLett.46.1220

Schierholz, R., Lacroix, B., Godinho, V., Caballero-Hernández, J., Duchamp, M., Fernández, A., 2015. STEM-EELS analysis reveals stable high-density He in nanopores of amorphous silicon coatings deposited by magnetron sputtering. Nanotechnology 26, 75703. https://doi.org/10.1088/0957-4484/26/7/075703

Schmidt, D., Kjerstad, A.C., Hofman, T., Skomski, R., Schubert, E., 2009. Optical, structural, and magnetic properties of cobalt nanostructure thin films. J. Appl. Phys. 105, 113508. https://doi.org/10.1063/1.3138809

Seydoux-Guillaume, A.-M., David, M.-L., Alix, K., Datas, L., Bingen, B., 2016. Trapping of helium in nano-bubbles in euxenite: positive identification and implications. Earth Planet. Sci. Lett. 448, 133-139. https://doi.org/10.1016/j.epsl.2016.05.013 
Snoeck, E., Majimel, J., Ruault, M.O., Hÿtch, M.J., 2006. Characterization of helium bubble size and faceting by electron holography. J. Appl. Phys. 100, 23519. https://doi.org/10.1063/1.2216791

Taverna, D., Kociak, M., Stéphan, O., Fabre, A., Finot, E., Décamps, B., Colliex, C., 2008. Probing physical properties of confined fluids within individual nanobubbles. Phys. Rev. Lett. 100, 35301. https://doi.org/10.1103/PhysRevLett.100.035301

Templier, C., 1991. Inert gas bubbles in metals: a review, in: Donnelly, S.E., Evans, J.H. (Eds.), Fundamental Aspects of Inert Gases in Solids. Springer, pp. 117132. https://doi.org/10.1007/978-1-4899-3680-6_10

Trinkaus, H., 1983. Energetics and formation kinetics of helium bubbles in metals. Radiat. Eff. 78, 189-211. https://doi.org/10.1080/00337578308207371

Walsh, C.A., Yuan, J., Brown, L.M., 2000. A procedure for measuring the helium density and pressure in nanometre-sized bubbles in irradiated materials using electron- energy-loss spectroscopy. Philos. Mag. A 80, 1507-1543. https://doi.org/10.1080/01418610008212134

Wilson, W.D., Bisson, C.L., Baskes, M.I., 1981. Self-trapping of helium in metals. Phys. Rev. B 24, 5616-5624. https://doi.org/10.1103/PhysRevB.24.5616 